\title{
Distributed Wireless Channel Allocation in Networks with Mobile Base Stations
}

\author{
Sanket Nesargi \\ Computer Science Program \\ University of Texas at Dallas \\ Richardson, TX 75083-0688. \\ e-mail: $\{$ sanket, ravip\}@utdallas.edu
}

\begin{abstract}
-
In traditional cellular systems with fixed base stations the channel reuse pattern is static and deterministic. When the cell layout is dynamic, due to the mobility of base stations, the cluster of cells within co-channel interference range changes with time. Consequently, the channel reuse pattern is highly dynamic. Moreover, base stations also need wireless channels to communicate amongst themselves. A communication session between a pair of nodes may have to switch channels due to the movement of other nodes into the neighborhood. Hence, there is a need for new wireless channel allocation algorithms for virtual cellular systems with mobile base stations. In this paper, principles of mutual exclusion pertaining to distributed computing systems are employed to develop such an algorithm. The interbase station wireless links are referred to as backbone links while the base station-mobile node links are referred to as short-hop links. The proposed algorithm is distributed, dynamic and deadlock free. Disjoint sets of channels are used for backbone and short-hop links. The distributed nature of the channel allocation scheme leads to robustness as the responsibility is no longer centralized at the MTSO. Instead, it is shared among all the mobile base stations. This also makes the algorithm scalable.
\end{abstract}

\section{INTRODUCTION}

Several existing models of mobile computing systems assume a cellular network of mobile nodes and stationary nodes [4]. The geographical area served by the system is divided into regions referred to as cells. Each cell has a fixed base station $(B S)$. A fixed wireline network connects all the base stations. The mobile nodes, referred to as mobile hosts ( $M H \mathrm{~s})$, present in a cell can communicate with other nodes in the system only through the $B S$ of that cell. This communication is through a wireless link between the $M H$ and its $B S$. There are two approaches to channel selection in such scenarios:

1. A centralized approach [3], [5], [8], [11], [21], [22] in which requests for channel allocation are forwarded to, and handled by, a central controller that has access to system wide channel usage information.

2. A distributed approach [1], [4], [9], [15] in which the base stations and/or the mobile hosts monitor the signal-to-noise ratio of relevant channels and, in some cases, exchange this information. The channel use decisions are made by each node, based on its information, without involving a central controller.

We deviate from the traditional cellular model and assume a virtual cellular network where the fixed base stations are replaced by mobile base stations $(M B S \mathrm{~s})$. The wireline links between the fixed $B S$ s are replaced by wireless links between $M B S \mathrm{~s}$. So, the entire network is wireless. The inter- $M B S$ links will, henceforth, be referred to as backbone links while the $M B S-M H$ links will be referred to as short-hop links. Figure 1 presents a logical view of the virtual cellular network.

The relative position of cells changes with time. As the relative positions of $M B S$ s cannot be determined a priori, it does not make sense to pre-allocate fixed sets of channels to each $M B S$ for short-hop links. Also, in the interest of scalability

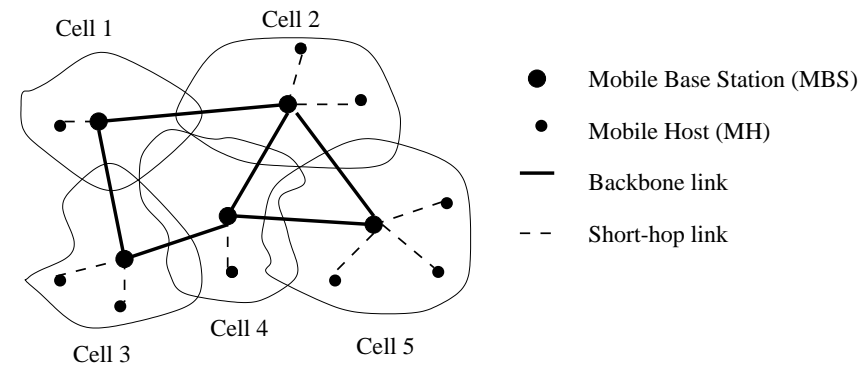

Fig. 1. A fully wireless cellular network.

and robustness a central controller should be replaced by a distributed channel allocation mechanism. Furthermore, it is our intention to minimize the amount of work that a resource poor $M H$ should have to perform for channel allocation. Instead, the responsibility is to be shared by all the $M B S \mathrm{~s}$ which are assumed to be comparatively resource rich, as described later in Section II.

We view the task of channel allocation as a form of the mutual exclusion problem studied extensively in the operating systems and distributed computing community. A mutual exclusion based algorithm for channel allocation in cellular networks was first presented by one of the authors in [15]. Here we modify and enhance the algorithm for systems with mobile base stations.

The set of wireless channels is partitioned into two disjoint subsets: one subset used exclusively for backbone links and another subset used exclusively for short-hop links. Channel partitioning simplifies the task of channel allocation at the cost of utilization. In the future we will extend our work to a scenario where all channels can be used for backbone as well as short-hop links. Preliminary ideas for such an extension have been presented in [13]. The proposed algorithm is flexible in the sense that it can also be used for traditional cellular systems just by setting the $M B S$ velocity to zero.

In simulation experiments, continuous tracking of all the nodes is computationally expensive. We present a simple approximation strategy in conjunction with discrete event simulation which significantly reduces the cost of simulations. At the same time, it models the mobility of $M B S \mathrm{~s}$ and $M H \mathrm{~s}$ with a degree of accuracy of the experimenter's choice. To ensure the correctness of the algorithm ${ }^{1}$ inspite of the approximations, the simulation experiments resort to a conservative channel allocation policy. Thus, simulation experiments using such models yield a conservative estimate of the performance of the algorithm.

\footnotetext{
${ }^{1}$ By correctness we mean avoidance of co-channel interference. 
The system model is described in Section II. The problem description is presented in Section III. The underlying theoretical basis for the algorithm, i.e., interpreting channel allocation as a mutual exclusion problem is described in Section IV. The algorithm and the proof of its correctness are presented in Section V. The cost saving simulation approximations are described in Section VI. Preliminary simulation results are presented in Section VII followed by the conclusion in Section VIII.

\section{SySTEM MODEL}

We assume a system consisting of a set of $M B S \mathrm{~s}$ and $M H \mathrm{~s}$ connected by a completely wireless network. The $M B S$ s have more resources than the $M H$ s in terms of energy supply, memory, processing power, etc. The $M B S$ s would be typically mounted on trucks, tanks, buses, etc. Neighboring $M B S$ s communicate using wireless backbone links forming a backbone network. No assumption is made about the mobility pattern of $M B S$ s. So, no guarantees are provided about the backbone network being connected all the time. Planning of $M B S$ mobility to avoid network partitioning is a separate problem that has been addressed in [18].

An $M H$ communicates only through a neighboring $M B S$. An $M H-M B S$ pair can establish a bidirectional short-hop wireless link provided their separation is less than a threshold value $d$. This is equivalent to an $M B S$ having a cell of radius $d$. If a wireless channel is being used to support a short-hop link between an $M H$ and an $M B S$, the same channel cannot be concurrently used to support any kind of communication within a radius $\alpha \times d$ around the involved $M B S$, where $\alpha>1$. Thus, the short-hop channel reuse distance is $\alpha \times d$.

Two $M B S$ s can establish a bidirectional wireless link provided their separation is no more than another threshold value $D$. If two $M B S$ s are using a channel for a backbone link, that channel cannot be simultaneously used to support any other communication in a region consisting of the union of two circles, each of radii $\beta \times D$ centered at the respective $M B S \mathrm{~s}$. Thus, $\beta \times D$ is the backbone channel reuse distance.

We assume that $D>d$. This is consistent with the earlier assumption that $M B S$ s have abundant energy supply enabling them to transmit at greater power levels than $M H \mathrm{~s}$. The variables $D, d, \alpha$ and $\beta$ are system parameters that depend on the networking hardware, power level of transmissions, fading characteristics, etc. Figure 2 presents a schematic representation of the co-channel interference ranges. Later, in Section VII, we specify the exact values of $\alpha$ and $\beta$ used for the simulation experiments.

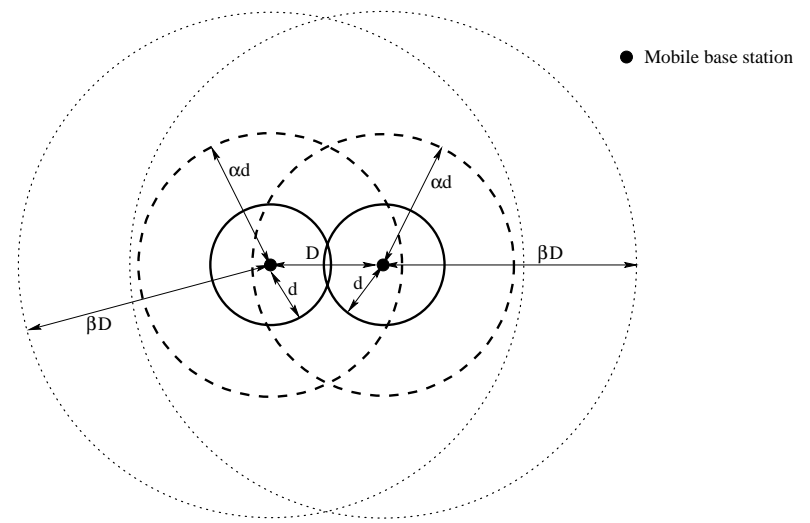

Fig. 2. Co-channel interference ranges for backbone and short-hop links.

The mobility of $M H \mathrm{~s}$ is modeled by the following three steps through which the $M H$ s loop:

1. When an $M H$ enters an $M B S$ 's cell, the $M H$ stays in that cell for a period of time determined by a probability distribution. 2. At the end of this period the $M H$ enters an arbitrarily selected neighboring cell. Note that due to the mobility of $M B S \mathrm{~s}$ the set of neighboring cells changes with time.

3. If there is no neighboring cell to migrate to, the $M H$ stays in the same cell as before. For modeling purposes this is treated just like an entry into a new cell. This ensures that the number of $M H \mathrm{~s}$ in the system remains unchanged.

Two realistic scenarios where such a mobility model would be applicable are the battlefield scenario and the public transport scenario.

In a battlefield, soldiers (equivalent to $M H \mathrm{~s}$ ) may move along with a tank (equivalent to an $M B S$ ) for a while before moving towards and connecting with a neighboring tank. This switch from one tank's cell to another would continue for the system lifetime.

Two kinds of wireless channels are available: communication channels and control channels. Communication channels are used to support backbone and short-hop links. Control channels are used to send messages generated by the channel allocation algorithm. We assume that the maximum range of a communication channel is equal to $D$, the range of a backbone link. However, control channel transmissions can be sent at a higher power level, if required, so that an $M B S$ can communicate with all the other $M B S \mathrm{~s}$ within the backbone co-channel interference range. Henceforth, when we use the term channel or wireless channel we mean communication channels. The wireless channels are assumed to be orthogonal to each other. So, only co-channel interference is considered.

\section{PROBlem Description AND CONTRIBUtions}

In cellular networks that have fixed cell layout, the cluster of cells that are within co-channel interference range do not change with time. Several fixed, dynamic, or hybrid channel allocation algorithms have been proposed for systems with fixed cell layout [1], [3], [4], [5], [6], [8], [9], [11], [15], [16], [21], [22]. When the base stations are mobile the cluster of cells within cochannel interference range changes with time, and the channel reuse pattern is highly dynamic. Existing algorithms are not designed to handle such dynamism. Hence, there is a need for new wireless channel allocation algorithms for systems with mobile base stations. The base stations also need wireless channels to communicate amongst themselves.

This raises some interesting issues:

1. Should the available frequency spectrum be divided into two disjoint sets of channels: a set of backbone channels and another set of $M B S-M H$ short-hop communication channels?

2. If the answer to the first question is in the affirmative, how many channels should be designated as backbone channels?

As the $M B S$ s move, their adjacency graph changes. Hence, the required number of backbone channels changes with time. Note that the separation of backbone channels from short-hop channels will simplify the channel allocation problem at the cost of efficiency of channel utilization. If the adjacency matrix of base stations is sparse, several backbone channels may be unutilized, and cannot be used for $M B S-M H$ communication either.

Concurrent presence of backbone and short-hop links with different signal strengths and range has some similarities with hierarchical cellular systems [20] having smaller microcells overlaid with larger umbrella cells. However, there are several important differences as well: (i) In hierarchical systems 
the relative configuration of microcells and umbrella cells remains unchanged. (ii) In hierarchical systems at least one node connected by a wireless link is fixed. However, in the proposed system both base stations connected by a backbone link may be moving. Hence, solutions for hierarchical cellular systems cannot be directly applied in the proposed scenario.

The problem at hand is to:

1. Develop a dynamic channel allocation algorithm for backbone as well as short-hop links.

2. Make channel allocation decisions in a distributed fashion to tide over the absence of any fixed central controller in a virtual cellular network, and to provide scalability and robustness.

3. Minimize the involvement of resource poor $\mathrm{MHs}$.

For simplicity we assume that the set of wireless channels is partitioned into two disjoint subsets: backbone channels and short-hop channels.

\section{Contributions of this paper}

A distributed, dynamic channel allocation algorithm is presented. The $M B S$ s make all the decisions based on the information available locally. An $M B S$ needs to exchange information with only its neighboring $M B S$ s within the co-channel interference range. Unlike the fixed channel allocation (FCA) algorithms, the proposed algorithm can adapt to changing load distribution in the network. It is more robust than existing $d y$ namic channel allocation (DCA) algorithms as it does not depend on a central network switch whose failure can bring down the entire network.

The salient features of the proposed algorithm are:

1. Bounded latency: No mobile node that wishes to acquire a wireless channel for a communication session is made to wait indefinitely before it is either allocated a channel or is informed of a failure to do so.

2. Deadlock freedom: There is no possibility of finding a set of mobile base stations involved in a circular wait while trying to satisfy channel allocation requests. So, the algorithm always makes progress.

3. Symmetry: All the $M B S$ s follow the same procedure for channel allocation. Hopefully, there will be no need to drastically redesign hardware, or develop new software if more $M B S$ s or $M H$ s are to be added.

4. Low system overhead and network traffic: As the proposed algorithm adapts to the temporal and spatial locality of load distribution, each new channel allocation request is handled with an exchange of zero or a small number of messages between the mobile base stations.

5. Concurrency: Requests for channel allocation originating independently and concurrently in different parts of the network can be processed simultaneously.

\section{THEORETICAL FRAMEWORK AND BASIC IDEA}

Mutual exclusion is an extensively studied problem in the fields of operating systems and distributed computing [2], [7], [10], [17], [19]. Multiple processes may be concurrently trying to access a shared resource. A process is said to be in its critical section during the time it has exclusive access to the shared resource.

\section{A. Channel Allocation vs. Mutual Exclusion}

In the context of a pair of communicating nodes $(M H-M B S$ or $M B S-M B S)$, the use of a particular channel to support a communication session is equivalent to a critical section execution by the communication session where the channel is the shared resource. Several neighboring cells may be concurrently trying to choose channels to support sessions in their region. This can lead to conflicts because the number of communication channels is limited. The resolution of such conflicts is similar to the mutual exclusion problem [2], [19]. The system has two distinct classes of shared resources: short-hop channels and backbone channels. Mutual exclusion for one class of resources is entirely independent of mutual exclusion for the other.

However, the channel allocation problem is more general than the mutual exclusion problem. First, an $M B S$ may be supporting multiple short-hop and/or backbone communication sessions, each session using a different communication channel. This is equivalent to an $M B S$ being in multiple, distinct critical sections concurrently. Second, existing mutual exclusion algorithms for distributed systems [2], [7], [10], [17], [19] assume that a node specifies the identity of the resource it wants to access in a critical section. Depending on the availability of that resource, appropriate decisions can be made. However, in distributed channel allocation, a cell asks for any channel as long as there is no co-channel interference. Due to the nonspecificity of the request and because neighboring mobile base stations make channel allocation decisions independently based on locally available information, the decision process becomes more difficult. Third, depending on whether a channel is being used for short-hop or backbone link, the co-channel interference range is different. This is quite different from the approach taken by distributed mutual exclusion models that make no distinction between who is using a resource or for what application: as long as a resource is being used by one process in the system, it cannot be used anywhere in the system.

Moreover, existing distributed mutual exclusion algorithms do not impose any upper bound on the elapsed time between a node's request for the resource and the granting of that resource. These algorithms are not suitable for the channel allocation problem which requires that decisions be made in realtime. So, a conservative approach that makes the channel allocation decisions quickly needs to be adopted. Such an approach may drop calls that a more general but time consuming approach would have supported. This is a trade-off that has to be accepted.

The mobility of base stations adds yet another degree of complexity to the problem. A short-hop channel may be concurrently assigned to two different short-hop sessions that are, initially, not in each other's co-channel interference range. However, due to the movement of the $M B S \mathrm{~s}$ and the $M H \mathrm{~s}$, during the lifetime of these communication sessions, the nodes involved in the two sessions may start approaching the co-channel interference region of each other. In order to avoid any conflict, at least one session will have to switch to another channel. A similar situation may arise for two backbone links that were initially far apart and were using the same channel. In the context of mutual exclusion, this is analogous to the pre-emption of the critical section execution of a node with a subsequent attempt to acquire another shared resource and enter a different critical section.

The channel allocation algorithm proposed in the next section is based on the ideas in the Ricart-Agrawala algorithm for mutual exclusion [17].

\section{B. Basic Idea}

An $M B S$ makes all channel allocation decisions on behalf of the $M H \mathrm{~s}$ in its cell. In some situations described in the next section, based on local information, an $M B S$ can assign a channel to a short-hop link between itself and an $M H$ without caus- 
ing any interference, and without having to consult the neighboring $M B S \mathrm{~s}$. Otherwise, requests time-stamped with Lamport's clock [7] are sent by the $M B S$ to neighboring $M B S \mathrm{~s}$ to determine the channel to be assigned for the short-hop link. As already mentioned in Section II, in the context of short-hop channel allocation, the list of neighboring $M B S$ s consists of all $M B S \mathrm{~s}$ within a distance of $\alpha \times d$ from the $M B S$ in question. The information received in the replies from the neighboring $M B S \mathrm{~s}$ is used to determine the channel to be allocated to the short-hop link. The distributed nature of the algorithm, and the finite but non-deterministic propagation delays of messages between $M B S$ s can lead to co-channel interference if a naive channel selection strategy is employed: multiple cells in each other's interference range may concurrently and independently decide to use the same channel for short-hop links in their cells. Such a possibility is prevented by associating a priority among concurrent requests based on their timestamps. As in the RicartAgrawala algorithm for mutual exclusion [17], an $M B S$ with a higher priority request defers replying to a lower priority channel allocation request.

Similarly, if channels have to be transferred from a lightly loaded cell ${ }^{2}$ to a heavily loaded neighbor, conflict is avoided in the following manner: having selected a communication channel for transfer, based on a round of message exchange with its neighbors, the mobile base station sends the channel identity to the neighboring mobile base stations. Only if all the neighboring mobile base stations approve of the selection is the channel transferred, otherwise not.

The set of channels allocated to a cell varies with time. Unlike existing DCA algorithms [21], [22], a newly acquired channel (acquired through transfer, for example) is not relinquished by a cell on completion of the communication session it was supporting in the cell. Instead, the channel remains allocated to the same cell until it has to be transferred to a neighboring cell. This enables the algorithm to adapt to temporal and spatial changes in load distribution. It also helps reduce the traffic due to channel allocation requests in the fixed wire network.

\section{Channel Allocation Algorithm}

The data structures and the strategy to allocate channels for short-hop links build upon those described in [15] for traditional cellular systems where the base stations are fixed. For the sake of completeness and readability the entire algorithm is presented instead of only presenting the differences from the algorithm presented in [15].

\section{A. Data Structures}

All the communication channels in the system are collectively represented by a set Spectrum. Spectrum is divided into two disjoint sets: Spectrum ${ }_{s}$ and Spectrum $_{b}$ for short-hop and backbone links, respectively. The channel with the lowest frequency band is considered to be the first channel and the channel with the highest frequency band is the $n^{t h}$ channel, where $n$ is

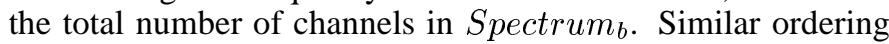
applies for channels in Spectrum . $_{\text {. }}$

The set of short-hop channels allocated to cell $C_{i}$ is represented by Allocate $_{s, i}$. Initially, Allocate $_{s, i}$ is an empty set for every cell $C_{i}$. The set of channels being used in a cell constitute its busy set. Unlike [15], the busy channels of cell $C_{i}$

\footnotetext{
${ }^{2}$ Cell load is the ratio of the number of short-hop channels the cell can use to set up links without any interference to the number of $M H$ s that are requesting such channels. Lower the demand to availability ratio, lower the load.
}

are distinguished into Busys, ${ }$ and Busy $y_{b, i}$ denoting the channels being used for short-hop and backbone links, respectively. For cell $C_{i}, B u s y_{s, i}$ is always a subset of Allocate $_{s, i}$. When a new short-hop communication request originates in $C_{i}$, one of the non-busy channels in Allocate $_{s, i}$ is assigned to support the communication session. If there is no such channel, then after a round of message exchange with the neighbors, a non-busy

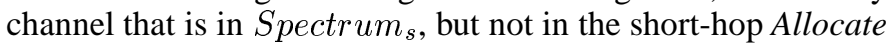
set of the cell or any of its neighbors is added to Allocate $_{s, i}$ as well as Busys,i. This channel is used to support the short-hop session. If such an attempt fails, $C_{i}$ tries to transfer a non-busy channel from the Allocate set of its neighbors to Allocate $_{s, i}$. If such a transfer is not possible, the communication request is dropped. Otherwise, the communication is successfully completed.

Also, cell $C_{i}$ maintains a transfer set, Transfer f $_{s, i}$, consisting of the channels earmarked as candidates for possible transfer from $C_{i}$ to one of its neighbors to support short-hop links involving their base stations. Transfer sets are initially empty for all the cells. All these sets are maintained by the corresponding mobile base stations.

Several communication requests may originate in a cell concurrently. These new requests may be ordered according to a policy decided a priori, like arrival time or $M H \_i d$. Only after the mobile base station has made a channel allocation decision about one locally originating request, does it process the next locally originating communication request in the sequence.

\section{B. Short-hop Channel Allocation}

(A) When a communication session is to be set-up in cell $C_{i}$, the following actions are taken by its mobile base station $\left(M B S_{i}\right)$ : 1. $T_{i} \leftarrow T_{i}+1$, where $T_{i}$ is the Lamport's clock at $M B S_{i}$;

2. $R T_{i} \leftarrow T_{i} / *$ time-stamp of this channel request. */

3. If $\left(\right.$ Available $_{s, i} \leftarrow$ Allocate $_{s, i}-$ Busy $_{s, i}-$ Transfer $_{s, i}$ ) $\neq \Phi$, then:

A highest order channel $k$ from Available $_{s, i}$ is selected to set-up the short-hop link;

Busy $_{s, i} \leftarrow$ Busys,$i \cup\{k\} ;$

Go to step 10;

else /* Available $_{s, i}=\Phi * /$

Send time-stamped REQUEST messages to each neighboring $M B S$ within short-hop co-channel interference range. ${ }^{3}$

4. When $M B S_{i}$ has received REPLY messages from each of its neighbors to which REQUESTs were sent, containing their short-hop Allocate, Busy and Transfer sets, it takes the union of Allocate $_{s, i}$ and the short-hop Allocate sets received in the REPLY messages, and stores the result in Interfere $_{s, i}$. Due to the mobility of $M B S \mathrm{~s}$ it is possible that an $M B S$ to which a REQUEST was sent has moved out of the co-channel interference range and can, or will, no longer send a REPLY. If $M B S_{i}$ does not receive a REPLY from $M B S_{j}$ within a timeout period $M B S_{i}$ assumes that: (i) $M B S_{j}$ has either crashed or moved out and does not send any subsequent messages to $M B S_{j}$, (ii) a reply has been received from $M B S_{j}$ indicating that its Allocate, Busy and Transfer sets are empty. Also, if $M B S_{k}$ that was hitherto out of short-hop co-channel interference range moves into the range during the execution of the channel allocation process, $M B S_{i}$ sends a REQUEST to $M B S_{k}$ and waits for its REPLY. 5. If $\left(\right.$ Free $_{s, i} \leftarrow$ Spectrum $_{s}-$ Interfere $\left._{s, i}\right) \neq \Phi$, then a channel of the highest order is selected from Free $_{s, i}$ and added

\footnotetext{
${ }^{3}$ The set of neighbors changes with time due to $M B S$ mobility. We assume that an $M B S$ knows the identity of neighboring $M B S$ s by listening to their beacons or by employing proximity determination protocols of the type described in [14].
} 
to Allocate $_{s, i}$. This channel is used to support the communication session. So, it is added to Busy $y_{s, i}$ as well. Then go to step 10.

6. If Free $_{s, i}=\Phi$, it does not mean that no channel is available for allocation. Perhaps, the communication session can be supported by transferring a channel. $M B S_{i}$ takes the union of Busy $_{s, i}$, Transfer $_{s, i}$, and the short-hop Busy and Transfer sets received in the REPLY messages in step 4, and stores the result in Inter fere $e_{s, i}$.

7. If $\left(\right.$ Free $_{s, i} \leftarrow$ Spectrum $_{s}-$ Interfere $\left._{s, i}\right)=\Phi$, then the short-hop channel request is dropped. Otherwise, the channel of the lowest order in Free $_{s, i}$ is chosen for the transfer.

8. Let the channel selected for transfer be $k$.

Busy $_{s, i} \leftarrow$ Busy $_{s, i} \cup\{k\}$;

Allocate $_{s, i} \leftarrow$ Allocate $_{s, i} \cup\{k\}$;

$M B S_{i}$ sends TRANSFER $(k)$ messages to all the neighbors in the co-channel interference range.

9. If all the neighboring cells to which the TRANSFER message was sent reply AGREED, then:

Channel $k$ is used to support the communication session;

$M B S_{i}$ sends RELEASE $(k)$ to all the neighboring cells;

Go to Step 10.

Otherwise: /* Some cells have sent REFUSE message. */

Allocate $_{s, i} \leftarrow$ Allocate $_{s, i}-\{k\}$;

Busy,$i_{\text {i }} \leftarrow$ Busys,$i-\{k\}$

$M B S_{i}$ sends $\operatorname{KEEP}(k)$ messages to the $M B S$ s of all the neighboring cells. $M B S_{i}$ selects the next channel from Fre $_{s, i}$, with order greater than that of $k$, and steps 8 and 9 are repeated. KEEP messages can be piggybacked on TRANSFER messages, if they are going to the same cell. To avoid excessive channel transfer overheads, under heavy load situations, the number of transfer attempts can be limited to the minimum of SHORT_HOP_THRESHOLD (parameter of the algorithm) and the cardinality of Free $_{s, i}$. If all attempts to transfer a channel fail, the communication request is dropped.

If $M B S_{i}$ does not receive an AGREED or REFUSE message from $M B S_{j}$ (to which the TRANSFER message was sent) within a timeout period, $M B S_{i}$ assumes that: (i) $M B S_{j}$ has crashed or moved out of co-channel interference range and is therefore of no consequence, (ii) an AGREED message is received from $M B S_{j}$.

10. Once a cell has decided to drop a request or to use a channel to support the corresponding communication session it sends all the deferred REPLYs to its neighbors.

11. When a short-hop communication session terminates in cell $C_{i}$, the corresponding channel is deleted from the set Busy $y_{s, i}$.

(B) When $M B S_{j}$ receives a REQUEST message from $M B S_{i}$ with timestamp $T_{i}$ :

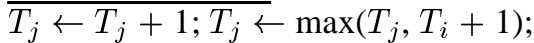

$M B S_{j}$ sends a REPLY message to $M B S_{i}$ if $M B S_{j}$ itself is not requesting a channel for a short-hop link, or if $M B S_{j}$ is requesting a channel and $M B S_{i}$ 's request's time-stamp is smaller than $M B S_{j}$ 's request's time-stamp (i.e., $T_{i}<R T_{j}$ or $T_{i}=R T_{j}$ and $i<j$ ). Otherwise, the REPLY is deferred. ${ }^{4}$ As $M B S_{i}$ only uses the union of the Busys,j and Transfer Tr, $_{s}$ sets received in the REPLYs, in Step $(A) .6$, and never uses the two sets separately, the communication overheads can be reduced by taking their union at $M B S_{j}$ and sending the result, rather than both the sets,

\footnotetext{
${ }^{4}$ The timeout duration in Step A.4 is large enough so that REPLY deferral does not cause timeouts.
}

in the REPLY message. Therefore, the REPLY message con-

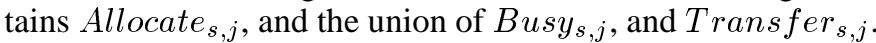

(C) When $M B S_{j}$ receives TRANSFER $(k)$ message from $M B S_{i}$ : if $k \notin$ Allocate $_{s, j}$ then send AGREED $(k)$ message to $M B S_{i}$. Otherwise, if $k \in$ Allocate $_{s, j}$ :

If $\left(k \in\right.$ Busy $\left._{s, j}\right)$ OR $\left(k \in\right.$ Transfer $\left._{s, j}\right)$ then send REFUSE $(k)$ message to $M B S_{i}$.

Otherwise

Transfer $_{s, j} \leftarrow$ Transfer $_{j} \cup\{k\}$;

Send AGREED $(k)$ message to $M B S_{i}$.

(D) When $M B S_{j}$ receives a RELEASE( $\left.k\right)$ message, the following actions take place:

Allocate $_{s, j} \leftarrow$ Allocate $_{s, j}-\{k\}$

Transfer $_{s, j} \leftarrow$ Transfer $_{s, j}-\{k\}$

(E) When $M B S_{j}$ receives $K E E P(k)$ message:

Transfer $_{s, j} \leftarrow$ Transfer $_{s, j}-\{k\}$

If, after sending an AGREED message to $M B S_{i}, M B S_{j}$ does not receive either a KEEP or a RELEASE message from $M B S_{i}$ within a timeout period, $M B S_{j}$ assumes that it has: (i) moved out of range of $M B S_{i}$, (ii) received a KEEP message from $M B S_{i}$ and acts accordingly.

\section{Backbone Channel Allocation}

Backbone channel allocation is similar to short-hop channel allocation, except for the following differences:

1. If a wireless channel is needed to establish a backbone link between two mobile base stations, $M B S_{i}$ and $M B S_{j}$, all the mobile base stations that are within a distance of $\beta \times D$ of at least one of $M B S_{i}$ and $M B S_{j}$ are polled to gather information about backbone channels in use in this region. This is consistent with the assumption made in Section II about the co-channel interference range of a backbone channel being $\beta \times D$.

2. There is no notion of allocate and transfer sets for backbone links. Let $M B S_{i}$ and $M B S_{j}$ be the mobile base stations between which a backbone link is to be established. REQUESTs are sent to all the $M B S$ s within the backbone cochannel interference range of this $M B S$ pair. The timestamp of the backbone channel requests is a tuple of the form $\left(\max \left(T_{i}, T_{j}\right), M B S_{i}, M B S_{j}\right)$. As in the short-hop channel allocation case, requests are prioritized by their timestamps: lexicographically lower timestamp indicates higher priority.

3. A mobile base station receiving the REQUEST immediately sends or defers the REPLY depending on whether (i) it is also involved in establishing a backbone link with a neighboring $M B S$, and (ii) the timestamp of its backbone channel request.

4. The REPLY sent by $M B S_{k}$ contains $B u s y_{b, k}$ : the set of backbone channels $M B S_{k}$ is currently using for its backbone links.

5. On receiving all the replies, the Inter fere ${ }_{b, i, j}$ set consists of the union of $B u s y_{b, i}, B u s y_{b, j}$ and the busy backbone sets received in the replies.

6. Free $_{b, i, j} \leftarrow$ Spectrum $_{b}-$ Inter fere $_{b, i, j}$.

If Free $_{b, i, j}=\Phi$, no backbone channel is available. Otherwise, the highest order channel is selected from Free $_{b, i, j}$ to establish a backbone link and that channel is added to $B u s y_{b, i}$ and Busy $_{b, j}$.

7. When a backbone link between $M B S_{i}$ and $M B S_{j}$ is broken, the channel that was being used to support the link is deleted from $B u s y_{b, i}$ and $B u s y_{b, j}$. 


\section{Channel Reconfiguration During Connection Lifetime}

There are two situations in which a link between two nodes may have to switch from one channel to another:

1. If an $M H$ moves out of one cell into another while involved in a communication session, handoff has to take place. As the problem has already been extensively studied, and existing solutions are applicable in the proposed system model, we skip the details of handoffs.

2. As stated earlier in Section IV, there is a possibility that a short-hop link between an $M B S-M H$ pair or a backbone link between a pairs of $M B S$ s may have to switch to a different channel during the lifetime of the corresponding session. This is not handoff as the pair of communicating nodes does not change. This switch from one channel to another is necessitated primarily by the mobility of $M B S \mathrm{~s}$ as described below.

Let there be a short-hop link between $M B S_{i}$ and $M H_{i}$ using a short-hop channel $i$. Concurrently, let there be another short-hop link in the network between $M B S_{j}$ and $M H_{j}$ also using channel $i$. Let the initial separation between $M B S_{i}$ and $M B S_{j}$ be greater than $\alpha \times d$, the short-hop co-channel interference range. At a later time, while the two short-hop sessions are still in progress, let one or both of $M B S_{i}$ and $M B S_{j}$ start moving towards the other. As stated earlier in the system model, the $M H$ s tend to move with the $M B S$ to which they are connected. When the separation between $M B S_{i}$ and $M B S_{j}$ becomes smaller than $\alpha \times d$ the two short-hop links, $M B S_{i}-M H_{i}$ and $M B S_{j}-M H_{j}$, using the same channel start interfering with each other. At least one of these links has to switch to another short-hop channel to avoid any further interference. Without loss of generality, let us assume that the $M B S_{i}-M H_{i}$ link has to switch to another short-hop channel. The procedure followed is equivalent to the termination of the old short-hop session between the node pair immediately followed by a new short-hop channel allocation between them.

Similarly, let us consider two backbone links: between mobile base station pairs $M B S_{i}-M B S_{j}$ and $M B S_{k}-M B S_{l}$. Both $M B S_{i}$ and $M B S_{j}$ are more than $\beta \times D$ distance away from $M B S_{k}$ and $M B S_{l}$. Both backbone links are using the same backbone channel without interfering with each other. Subsequently, if these nodes move so as to be within the backbone co-channel interference range of each other, at least one link will have to switch to another channel.

The responsibility for channel reconfiguration lies with the mobile base stations. When the hitherto far apart $M B S_{i}$ and $M B S_{j}$ detect that they have moved within distance $\beta \times D$ of each other, they exchange information about their backbone channel usage and make the appropriate channel reconfiguration decisions. When $M B S_{i}$ and $M B S_{j}$ detect that they have moved to within $\alpha \times d$ of each other, they exchange information about their short-hop channel usage and determine if any shorthop links need to be switched to other channels. We assume that the underlying MAC sub-layer and network layer protocols for node beacons along with timestamps and location stamps enable $M B S$ s to determine their distance from each other. These protocols are described in [14].

\section{E. Properties}

Now, we state some properties of the algorithm without proof. For a proof of these properties please refer to the U.T. Dallas technical report [12].

Lemma 1: The channel allocation algorithm avoids cochannel interference.
Lemma 2: Each new request for a communication session originating in a cell $C B_{i}$ causes a finite number of messages to be exchanged between the mobile base stations of the cell and its neighbors: $5 N \leq$ messages needed to make a channel allocation decision $\leq 2 N+3 N \times$ minimum $\left(\mid\right.$ Free $_{i} \mid$ , SHORT_HOP_THRESHOLD), where $N$ is the number of neighboring cells in the short-hop co-channel interference range.

Lemma 3: The channel allocation algorithm is deadlock free.

\section{PARAmeter Modifications FOR Simulation SPEEDUP}

In Section V-D it was mentioned that mobile base stations need to keep track of the $M B S \mathrm{~s}$ in their proximity for the purpose of channel reconfiguration and to avoid co-channel interference. This would require continuous tracking of the separation between pairs of mobile base stations making the simulation very expensive. Moreover, there may also be need for frequent channel reconfigurations which is an expensive operation. This has the potential to slow down the simulation.

A means to increase the speed of the simulation experiments would be to perform channel reconfigurations at fixed time intervals. However, a naive implementation of such a policy may lead to co-channel interference, thus violating the correctness of the simulation experiments. Therefore, we propose to employ a conservative simulation strategy with the following properties:

1. The short-hop link establishment/break process can be initiated as soon as the $M H$ makes the request for such a link (call arrival) or sends a disconnect message.

2 . Inter- $M B S$ distances are measured at fixed intervals of time, and it is only at these times that backbone link establishment and tear-down as well as channel reconfigurations for short-hop and backbone links are performed.

The cost of simulation speedup is reduced channel utilization, as will become obvious shortly.

Let the inter-reconfiguration interval be $t$, and let the upper bound on the speed of $M B S$ s be $s$. Then, the distance an $M B S$ could have moved during the interval is bound by $s \times t$.

When allocating a channel for a short-hop link between an $M H$ and $M B S_{i}, M B S_{i}$ considers all $M B S$ s within distance $\alpha d+2 s t$ to be within its short-hop co-channel interference range. Let the short-hop channel selected to establish the link be channel $i$. This ensures that at the time of this short-hop link establishment the minimum separation between $M B S_{i}$ and another mobile base station $M B S_{j}$ using channel $i$ at the same time is $\alpha d+2 s t$. If until the next reconfiguration time both $M B S_{i}$ and $M B S_{j}$ move directly towards each other at the maximum speed $s$ they cannot get any closer than $\alpha \times d$ until reconfiguration is performed, at which time one of the short-hop links will be made to switch to another channel.

Similarly, a backbone link is established between two $M B S$ s provided their separation is no more than $D-2 s t$, instead of $D$ as stated earlier. Also, the backbone co-channel interference range is considered to be equal to $\beta D+2 s t$, i.e., every base station within this distance of at least one of the two $M B S \mathrm{~s}$ is polled. As a result, even if the two $M B S$ s participating in the backbone link keep moving directly away from each other, by the next channel reconfiguration occurrence, their separation from each other will be no more than $D$, i.e., they will still be in backbone range. Also, all other $M B S$ s using the same backbone channel will be at least $\beta \times D$ away from these two $M B S$ s until the next reconfiguration. 


\section{Simulation EXPERIMENTS AND RESUlts}

We conducted simulation experiments to evaluate the performance of the proposed algorithm. We assumed a system composed of 100 mobile base stations and 1000 mobile hosts. All the nodes in the system were always located within a square of side 15 kilometer. Initially, the mobile base stations were uniformly distributed within the square with the immediate neighbors along the $x$ - and $y$ - axes at distance of 1.5 kilometer from each other.

The mobile base stations moved in a random fashion in the square at a constant speed, $s$, of 10 kilometer per hour. Every $M B S$ randomly chose any point in the square as its next destination and moved towards it until it got there. Then, it chose the next point and moved towards it, and so on. This was repeated until the end of the simulation. Initially, each $M H$ was associated with an $M B S$ in whose cell it was resident and an equal number of $M H \mathrm{~s}$ were associated with each $M B S$. The $M H$ s moved with the $M B S$ with which they were associated. The time an $M H$ spent in an $M B S$ 's cell before moving into a neighboring $M B S$ 's cell was exponentially distributed with a mean of 30 minutes. If the old $M B S$ did not have any neighbor, the $M H$ continued to stay in the same cell for a period of time obtained from the distribution mentioned above.

The length of a short-hop communication session (also referred to as a call) was exponentially distributed with a mean of 3 minutes. For a given $M H$ the time between two successive short-hop channel requests (the reciprocal of the call arrival rate) was also exponentially distributed and the mean value was varied to simulate various levels of channel demand.

We assumed that the number of backbone channels was large enough so that no backbone link establishment was prevented for lack of a channel. We made this assumption because the purpose of our experiments was to study the impact of mobility and channel demand on short-hop channel allocation. However, in our simulation experiments we did measure the number of backbone channels being used to get an idea of how many such channels are really needed. We also varied the total number of short-hop channels in the system from 20 to 100 .

We assumed that the range of a cell $(d)$ is 1 kilometer. In the initial configuration the entire $15 \mathrm{~km} \times 15 \mathrm{~km}$ square is served by at least one $M B S$ with some overlap between neighboring cells. Two $M B S$ s can have a backbone link between them provided they are not more than $2-2 s t$ kilometer apart, i.e., $D$ is equal to 2 kilometer. The values of $\alpha$ and $\beta$ were set to 4 and 3, respectively. So, if an $M B S$ is using a channel for a short-hop link, the same channel can be used by another $M B S$ to support a short-hop link provided the other $M B S$ is at least $4 d=4$ kilometer away. Also, two $M H$ s concurrently using the same channel for their respective short-hop links can never be less than 2 kilometer from each other, thus avoiding co-channel interference. The same channel can be used to concurrently support two backbone links provided both $M B S \mathrm{~s}$ in one pair are at least $3 D=6$ kilometer away from both $M B S \mathrm{~s}$ in the other pair.

In each run of the simulation, no data collection was performed until the first 15,000 calls were completed. This was done to filter out the impact of any initial transient effects. Then, data collection was performed until the next 50,000 calls were either completed or dropped due to lack of channels.

We conducted experiments described below. For each experiment, the following values were measured: (i) percentage of dropped calls due to non-availability of short-hop channels, (ii) average number of channel allocation messages sent per call, (iii) number of short-hop links reconfigured during each reconfiguration stage, (iv) average number of backbone links in existence during the simulation period, and (v) average number of backbone channels in use during the simulation period.

\section{A. Experiment I: Short-hop Link Characteristics}

In this experiment the short-hop call arrival rate and the number of channels in the short-hop spectrum, Spectrum S $_{s}$, were varied. Their impact on the number of dropped calls and the number of successful handoffs was studied. Calls that could not be connected initially, or were discontinued at the time of handoff or reconfigurations due to non-availability of channels are all counted as dropped calls. The simulation results are shown in Figure 3. For these experiments the inter-reconfiguration interval is set to 2 minutes.
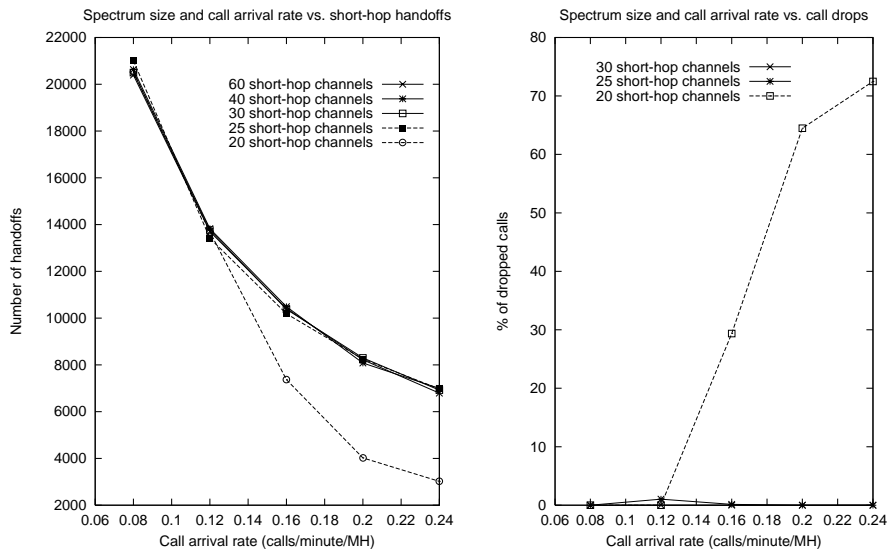

Fig. 3. Impact of spectrum size and call arrival rate on the number of handoffs and call drops.

When the call arrival rate is low, no call is dropped as it is possible to find an available channel from Spectrum S $_{\text {. Also, due }}$ to the availability of short-hop channels all the calls in progress can be handed-off when the corresponding $M H \mathrm{~s}$ move from one $M B S$ to another. Hence, the number of handoffs is also high. However, as the call arrival rate increases, for a given size of Spectrum $_{s}$, the availability of free channels decreases and so does the number of handoffs. The decrease is sharper for smaller Spectrum $_{s}$ sizes. Beyond a point, calls start getting dropped. With further increase in the call arrival rate, the percentage of dropped calls increases steadily. Obviously, for a given call arrival rate, the greater the size of Spectrum $_{s}$ the higher the availability of free short-hop channels and lower the percentage of dropped calls.

We observed that the number of short-hop handoffs decreases with increasing call arrival rate. Due to high channel demand at high call arrival rates an $M H$ 's request for a channel in the new cell is less likely to succeed. For larger Spectrums sizes the likelihood of a channel request being satisfied during the initial request, and, subsequently, a handoff attempt also succeeding are higher. This leads to a greater number of successful handoffs

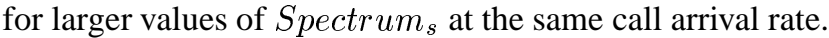

The impact of call arrival rate and the size of Spectrum $_{s}$ on the number of messages required to establish and maintain

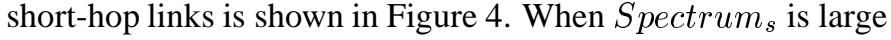
and/or arrival rate is low, the number of short-hop messages needed to allocate a channel is small, and remains almost unchanged. Also, note that the number of short-hop messages in such situations is considerably smaller than the expected number of $M B S$ s within short-hop co-channel interference range. 


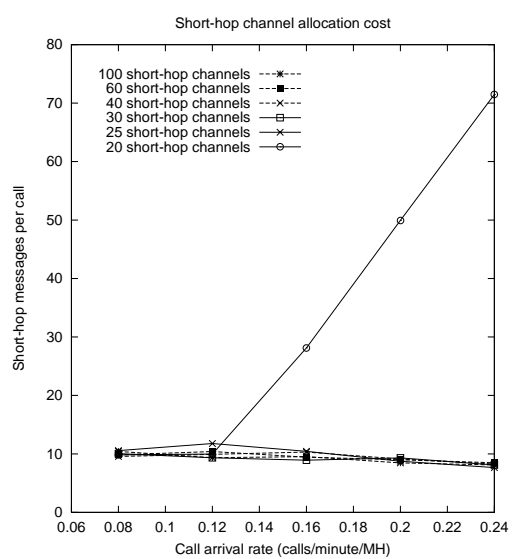

Fig. 4. Impact of spectrum size and call arrival rate on the cost of maintaining short-hop links.

This is because when a short-hop channel request arrives at an $M B S$, with high probability the $M B S$ finds that it has a channel in its Allocate set that is not busy. So, the channel request can be satisfied locally without having to send any REQUESTs to the neighboring $M B S \mathrm{~s}$. Even if there is no free channel in the Allocate set, our experiments indicate that the channel request can be satisfied in one round of REQUEST and REPLY exchanges, with negligible instances when channel transfers are required.

However, for smaller sizes of Spectrum $_{s}$, and beyond a certain call arrival rate, the likelihood of finding a free channel diminishes. So, a larger fraction of call arrivals result in the exchange of REQUESTs and REPLYs, and even in channel transfers. So, the number of short-hop messages per call increases with the rate of call arrival. This is evident in the figure when only twenty short-hop channels are available.

Most of the time a system will operate in situations of low to moderate load. In such operating conditions the number of messages exchanged for channel allocation will be small. So, the proposed algorithm will incur low overheads. Note that we have made no assumptions about the mobility pattern of $M B S \mathrm{~s}$. So, there will be periods when several $M B S$ s can come close to each other requiring a larger number of messages to be exchanged per call. During periods when the $M B S \mathrm{~s}$ are far apart, there will be fewer $M B S \mathrm{~s}$ in a given $M B S$ 's short-hop cochannel interference range leading to fewer messages. The numbers reported here are long term averages which even out the temporal variations.

\section{B. Experiment II: Impact of Reconfiguration Interval}

First, we measured the number of short-hop links that have to be assigned a different channel at the time of reconfiguration, with the reconfiguration interval equal to 2 minutes. Then, we also measured the impact of varying the reconfiguration interval on the number of backbone links and the cost of setting up such links. The simulation results are shown in Figure 5.

Impact on number of channel rearrangements: During channel reconfiguration (which happens once every two minutes), the number of calls in progress is small if the call arrival rate is low. Hence, the number of short-hop links requiring channel rearrangements at the time of reconfiguration is also small.

As the call arrival rate increases more calls are in progress at the time of reconfiguration. There is a higher probability of two short-hop links, using the same channel, to come within each other's interference range due to the movement of $M B S \mathrm{~s}$, and
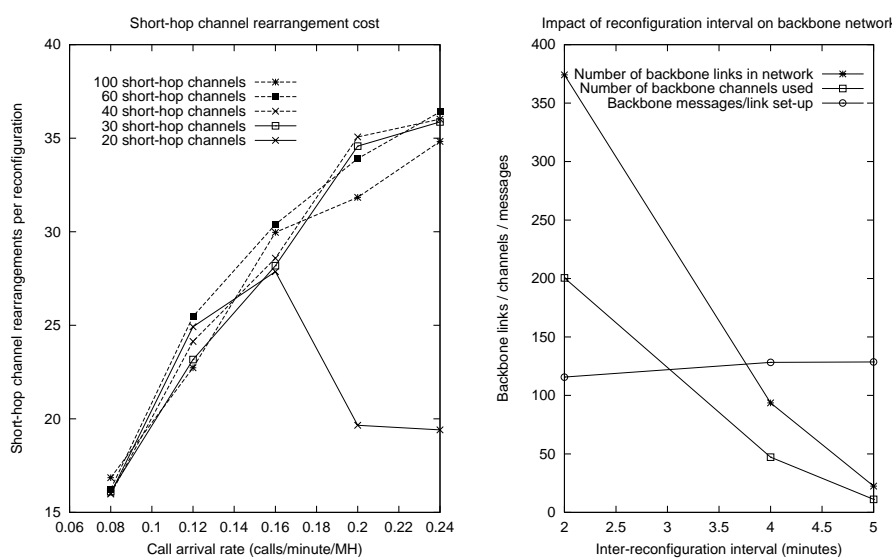

Fig. 5. Impact of channel reconfigurations on short-hop and backbone links.

the movement of $M H \mathrm{~s}$ along with them. Hence, the number of channel rearrangements required during the reconfiguration time also increases and channels are available for such rearrangement. This increase in rearrangements is observed only upto a certain call arrival rate. We will refer to this threshold call arrival rate as the knee of the curve. Beyond the knee, the number of rearrangements decreases with increasing call arrival rate. We believe that this is due to two reasons:

1. At higher arrival rates several calls get dropped right in the beginning due to the non-availability of channels, thus leaving fewer short-hop links that can potentially require channel rearrangements.

2. At the time of reconfiguration, interfering short-hop links may be unable to find free channels to switch to. Thus the number of successful reconfigurations decreases. This is consistent with the increase in the number of dropped calls at higher call arrival rates, as shown in Figure 3.

Greater the size of Spectrum $_{s}$, higher the call arrival rate at which the knee is reached. For example, in Figure 5, the knee is reached for a twenty short-hop channel system when the call arrival rate is close to 0.16 . The curves for systems with larger number of short-hop channels start flattening around 0.22 to 0.24 , indicating that they are reaching their respective knees.

Impact on backbone links: The cost of setting up backbone links and their number is independent of the number of shorthop links and the short-hop call arrival rate. In our simulation the cost depends on the time between successive reconfigurations. This is because we add an extra $2 s t$ distance to the backbone co-channel interference range $(\beta D)$ to ensure that if two backbone links do not interfere at the time of reconfiguration they also do not interfere until the next reconfiguration. Hence, greater the interval between successive reconfigurations, greater the virtual co-channel interference range. As backbone allocation messages have to be exchanged with all $M B S \mathrm{~s}$ within the virtual backbone co-channel interference range, the number of such messages inceases with an increase in the interreconfiguation interval $(t)$ as shown in Figure 5.

Also, a backbone link can be established between two $M B S \mathrm{~s}$ only if the distance between them is less than $D-2 s t$. Therefore, as the value of $t$ increases, the number of $M B S$ s pairs that are eligible for backbone links reduces. This leads to a reduction in the number of backbone links and the number of backbone channels used as shown in Figure 5.

When the reconfiguration interval is equal to 2 minutes, backbone links can be established between two $M B S$ s provided they are within $D-2 s t$, i.e., 1.33 kilometer of each other. Also, each 
participating $M B S$ 's co-channel interference region is a circle of radius 6.67 kilometers and area at most 140 square kilometers. Actually, for $M B S \mathrm{~s}$ that lie closer to the edges of the simulated area, the co-channel interference area is smaller. Our approximate calculations yielded the average co-channel interference region of a backbone link to be about 120 square kilometers resulting in a backbone channel reuse factor of 2 . This is consistent with the simulation results where the number of backbone links is approximately double the number of backbone channels used. As the geographical area covered by the system increases, the reuse factor will also increase.

\section{CONCLUSION}

A mobile computing system with no fixed nodes was presented. The problem of concurrently allocating channels for backbone as well as short-hop links was formalized. Wireless channel allocation was interpreted as a variation of the mutually exclusive resource allocation problem.

A distributed dynamic channel allocation algorithm for systems with mobile base stations was presented. The algorithm built on the ideas first presented in the Ricart-Agrawala mutual exclusion solution. Disjoint sets of wireless channels were used for backbone and short-hop links. This policy was adopted to simplify the channel allocation process. The algorithm imposed only modest communication overheads. The percentage of dropped calls is negligible for low and moderate load conditions. Due to node mobility, communication sessions using a particular channel without any interference may start experiencing interference at a later time. So, channel reassignment is very important to avoid co-channel interference. Our simulation experiments showed that the overheads imposed by channel rearrangement are small.

The proposed algorithm can be used, without any modification, in cellular systems with fixed base stations. Afterall a cellular network is an instance of the network described in this paper: (i) the speed of the base stations is zero, (ii) backbone links do not need wireless channels. The algorithm is scalable as the control is distributed among $M B S \mathrm{~s}$.

We propose to extend our work to an algorithm in which every channel can be used to support backbone and short-hop communication sessions. Such a generalization will improve the utilization of channels, but also increase the complexity of the channel allocation process. We also intend to simulate the performance of the proposed algorithm for larger geographical areas, a greater number of nodes, and a larger number of calls. Furthermore, we also intend to measure the variations in the algorithm's performance with time as $M B S \mathrm{~s}$ converge towards or diverge from small regions in the system area.

\section{Acknowledgments}

The authors thank S. Venkatesan, Kartik Chandran and all the other members of the Distributed Systems Laboratory for invaluable advice and stimulating discussions.

\section{REFERENCES}

[1] Y. Akaiwa and H. Andoh. Channel Segregation - A Self-Organized Dynamic Channel Allocation Method: Application to TDMA/FDMA Microcellular System. IEEE Journal on Selected Areas in Communications, 11(6):949-954, August 1993.

[2] K. M. Chandy and J. Misra. The Drinking Philosophers Problem. ACM Transactions on Programming Languages and Systems, 6(4):632-646, October 1984.

[3] J. C.-I. Chuang. Performance Issues and Algorithms for Dynamic Channel Assignment. IEEE Journal on Selected Areas in Communications, 11(6):955-963, August 1993.
[4] L. J. Cimini, Jr., G. J. Foschini, and C.-L. I. Distributed Dynamic Channel Allocation Algorithms for Microcellular Systems. In J.M. Holtzman and D.J. Goodman, editors, Wireless Communications - Future Directions, pages 219-241. Kluwer Academic Publishers, 1993.

[5] S. A. El-Dolil, W. C. Wong, and R. Steele. Teletraffic Performance of Highway Microcells with Overlay Macrocell. IEEE Journal on Selected Areas in Communications, 7:71-78, January 1989.

[6] T. J. Kahwa and N. D. Georganas. A Hybrid Channel Assignment Scheme in Large Scale, Cellular Structured Mobile Communication Systems. IEEE Transactions on Communication, 26(4), April 1978.

[7] L. Lamport. Time, Clocks and the Ordering of Events in a Distributed System. Communications of the ACM, 21(7):558-565, July 1978

[8] W. C. Y. Lee. New Cellular Schemes for Spectral Efficiency. IEEE Transactions on Vehicular Technology, VT-36, November 1987.

[9] K. Madani and H. A. Aghvami. Performance of Distributed Control Channel Allocation (DCCA) Under Non-Uniform Traffic Condition in Microcellular Radio Communications. In Proceedings of the IEEE International Conference on Communications, pages 206-210, 1994.

[10] M. Maekawa. A $\sqrt{N}$ Algorithm for Mutual Exclusion in Decentralized Systems. ACM Transactions on Computer Systems, pages 145-159, May 1985.

[11] R. Mathar and J. Mattfeldt. Channel Assignment in Cellular Radio Networks. IEEE Transactions on Vehicular Technology, 42(4):647-656, November 1993.

[12] S. Nesargi and R. Prakash. Distributed Wireless Channel Allocation in Networks with Mobile Base Stations. U.T. Dallas, Computer Science Technical Report, 1998 (also available from www.utdallas.edu/ ravip/paper.list.html)

[13] R. Prakash. Distributed Wireless Channel Allocation in Cellular Systems with Mobile Base Stations. In Workshop on Nomadic Computing, Geneva, April 1997.

[14] R. Prakash and R. Baldoni. Architecture for Group Communication in Mobile Systems. In Proceedings on the IEEE Symposium on Reliable Distributed Systems (to appear), October 1998.

[15] R. Prakash, N. Shivaratri, and M. Singhal. Distributed Dynamic Channel Allocation for Mobile Computing. In Proceedings of the $14^{t h} A C M$ Symposium on Principles of Distributed Computing, pages 47-56, Ottawa, Canada, August 1995.

[16] S. S. Rappaport. The Multiple-Call Hand-Off Problem in High-Capacity Cellular Communications Systems. IEEE Transactions on Vehicular Technology, 40(3):546-557, August 1991.

[17] G. Ricart and A. K. Agrawala. An Optimal Algorithm for Mutual Exclusion in Computer Networks. Communications of the ACM, 24(1):9-17, January 1981.

[18] C. Shields, Jr., V. Jain, S. Ntafos, R. Prakash, and S. Venkatesan. FaultTolerant Mobility Planning for Rapidly Deployable Wireless Networks. In Proceedings of the IEEE Workshop on Fault-Tolerant Parallel and Distributed Systems, LNCS. Springer-Verlag, April 1998.

[19] M. Singhal. A Dynamic Information-Structure Mutual Exclusion Algorithm for Distributed Systems. IEEE Transactions on Parallel and Distributed Systems, 3(1):121-125, January 1992.

[20] C. W. Sung and W. S. Wong. User Speed Estimation and Dynamic Channel Allocation in Hierarchical Cellular System. In Proceedings of the $44^{\text {th }}$ Vehicular Technology Conference, pages 91-95. IEEE, 1994.

[21] M. Zhang and T.-S. P. Yum. Comparisons of Channel-Assignment Strategies in Cellular Mobile Telephone Systems. IEEE Transactions on Vehicular Technology, 38(4):211-215, November 1989.

[22] M. Zhang and T.-S. P. Yum. The Nonuniform Compact Pattern Allocation Algorithm for Cellular Mobile Systems. IEEE Transactions on Vehicular Technology, 40(2):387-391, May 1991. 